\title{
Autonomous navigation of robotic units in mobile sensor network
}

\author{
Sedat Nazlibilek \\ Atilim University, Department of Mechatronics Engineering, Ankara, Turkey \\ Bilkent University, Nano Technology Research Center - NANOTAM, 06800 Ankara, Turkey
}

\section{A R T I C L E I N F O}

\section{Article history:}

Received 11 June 2011

Received in revised form 15 October 2011

Accepted 31 January 2012

Available online 15 February 2012

\section{Keywords:}

Mobile robots

Sensor networks

Magnetic anomaly

Navigation

Positioning

Localization

\begin{abstract}
A B S T R A C T
This work is motivated by the problem of detecting buried anti-tank and anti-personnel mines in roads or some border regions. The problem is tried to be solved by use of small mobile robotic sensors and their some abilities such as measurement of local fields, navigation around a region, communications with each other, and constituting team within a mission area. The aim of this work is to investigate the navigation problem for the team behavior of mobile sensors within a potential field available in a small-scale environment such as an indoor area or an outdoor region. The mobile sensor network here is a collection of robotic units with sensing capability of earth magnetic field anomalies. A new kind of positioning system is needed for their collective behavior. In this work, a new method of navigation is proposed as a local positioning system. It utilizes ultrasound and radio frequency information to determine the coordinates of the points inside the operational area. The method proposed here is compared with the ultra wideband ranging ping-pong method that is used widely in recent applications. A time division multiple access method is used for the communications among the mobile sensors. The results on the positioning methods together with several simulations and experimental works are given. It is shown that the positioning method utilizing ultrasound-radio frequency method can give fairly good results.
\end{abstract}

(c) 2012 Elsevier Ltd. All rights reserved.

\section{Introduction}

This work is motivated by the problem of detecting buried anti-tank and anti-personnel mines in roads or some border regions. The mines had been buried mainly for border protection. In recent years, governments desire to clean these regions in order to open them for agriculture. "Clearing minefields is a long, slow, time-consuming process, and there is no room for error," said Capt. Jonathan Zimmer, the assistant officer in charge of the Mine Action Center. "We go by NATO standards, ... which means we have to use two types of methods to clear a minefield and must obtain 99.6\% clear." There are several methods to clean mine fields such as using metal detectors, animals and ground penetrating radars. There are advantages and disadvan-

E-mail address: nazlibileksedat@yahoo.com tages of these methods. Comparison of techniques is out of the scope of this paper.

We use a method making use of the information gathered by the earths' magnetic anomalies created by buried objects [1]. We try to develop a system that can detect anti-tank (AT) and anti-personnel (AP) mines in a minefield. The system used here is a mobile sensor network. It is composed of mobile sensor units with the capability of detecting magnetic anomalies by KMZ51 magneto-resistive sensor mechanism. The mobile unit determining the direction of the gradient vector moves along the direction of steepest descent of magnetic anomalies (Fig. 1). The sensor mechanism is a $3 \times 3 \mathrm{KMZ51}$ sensor grid acting as a convolution mask that is used to determine the gradient vector of the magnetic field anomaly variation at its location. During the course of action, they need to know their coordinates of locations and to communicate them with the members of the network. Therefore, we want to 


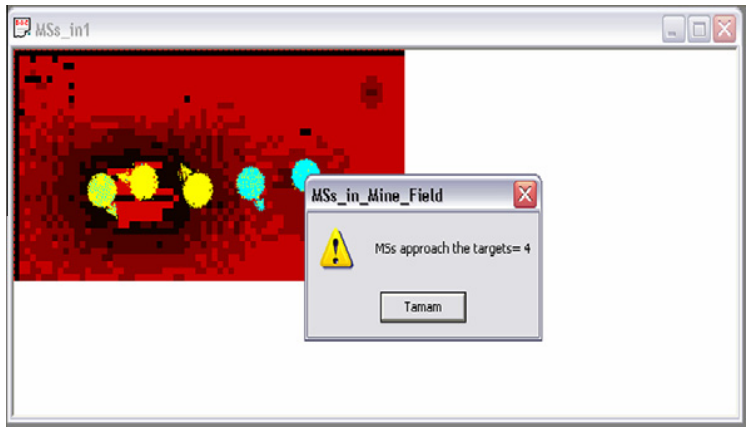

Fig. 1. The mobile sensor network detecting magnetic anomalies created by buried objects such as anti-tank, anti-personnel mines or any other objects.

develop a navigation capability by which the robots would determine their locations. The term "navigation" is defined as the determination of the coordinates of mobile sensors by themselves using an external reference positioning system.

In our application, a minefield is divided into longitudinal stripes. One of the stripes is about $1.5 \mathrm{~m}$ in width and the size of the area scanned at a time is about 1.5 $\times 1.5 \mathrm{~m}$ in dimensions. The Navstar GPS is not suitable for small-scale applications mentioned here. It cannot give the location within the scale of centimeters. A new kind of positioning system is needed. We try to develop a local positioning system fitting our purpose.

In recent years, a great deal of effort has been assigned to solve this type of positioning, localization or ranging problem in small scale environments. There are a wide range of methods and applications in this field. One of the emerging technologies in this area is the use of ultra wideband (UWB) radios for solving localization problem within indoor and small scale outdoor applications [2]. The other technological means used for ranging and positioning is the ultrasound (US) sensors [3,4]. Almost all of the robotic systems might utilize US sensors. Using ultrasound to measure distances in a sensor network has been extensively studied in previous works [5].

In our work, we apply a method that utilizes both ultrasound (US) and radio frequency (RF) information to determine the coordinates of the points inside the operational area. The method is adapted from [8] where a virtual ruler is moving around the fixed sensors randomly and the distances between the sensors are determined with respect to the virtual ruler. However, in contrast to moving the reference points around the region, in our study, a fixed coordinate reference frame is established within the region. The origin of the reference frame is also serves as the broadcast station of the sensor network. It carries the US and RF transmission systems on them. The mobile sensors moving over the region, receive the US and RF transmissions broadcasted simultaneously by the broadcast stations in a predetermined sequence. They calculate their coordinates with reference to the coordinate frame. In most of the cases, the distance measurements are done in a lineof-sight condition. We use a similar approach as the Cricket system [6], which attaches two ultrasound transceivers to the MICA2 nodes developed by UC Berkeley [7]. The Cricket sensor nodes broadcast the radio signals and ultrasonic signals at the same time. The ultrasonic signals will reach a receiving node later than the radio signals due to their speed difference. By measuring the flight delay of the ultrasonic signals, the distance between sender and receiver can be estimated based on the constant speed of ultrasonic signals.

The performance of our ranging system is compared with the ultra-wideband (UWB) Indoor Ranging System described in [9]. The details of mathematically modeling impulse radio are given in [10]. Evaluation of an UWB propagation channel is discussed in [11]. A time-of-arrival (ToA) based ranging scheme using UWB radio link is proposed in [12]. We utilize this proposed approach for the comparison with US-RF approach. UWB transmission is considered best for low-power indoor or small scale outdoor applications where there is high clutter, that is, the surrounding environment causes significant amount of multipath. The advantage of impulse radio is that it has a very simple transmitter and receiver design. Transmission is performed at baseband. In addition, impulse radio has the capability to provide very high data rates over short distances. It mitigates the effects of multipath. The multipath differential delay or delay spread is on the order of nanoseconds [13]. The new local positioning system which is called US-RF navigation system has two functions: the first one is the creation of a reference coordinate frame and the second one is to provide a means to the mobile sensors for locating their coordinates within this reference frame.

The navigation is needed for two reasons. One is for the central control/coordination station to know the coordinates of the mobile sensors, and the other is for the mobile sensors to know the coordinates of themselves and the team members in order to achieve a team behavior such as flocking in the region of operation. The former is needed for monitoring purposes of the mobile sensors and the latter is needed for team behavior of the mobile sensors. Team behavior of mobile sensors depends on two factors. One is the availability of a potential field distributed over the region and the other is the information of coordinates of mobile sensors acting in the region [15-22]. Mobile sensors within an operational area have to communicate with each other. They have to send their location information to the members of the team. A time division multiple access (TDMA) method is used for the communications, although it can limit the maximum number of mobile sensors used in a region. The synchronization among the mobile sensors can also be achieved by the TDMA method applied here. Each robot can move only within its time slot by making necessary rotation and translation.

The paper is organized as follows. The Section 2 gives the navigation methods. In this section, the system architecture is described and the methods for coordinate determination namely the US-RF method proposed here and the UWB method for performance comparison purposes are given. This section also presents comparative results for the two methods. Team constitution and behavior is given in Section 3. The detection process, simulations and practical applications and experiments carried out in the region 
where magnetic anomalies exist are given in Section 4. A general conclusion and future work are discussed in Section 5 .

\section{Navigation methods}

There are two methods for navigation in this application, namely ultrasound-radio frequency method and ultra-wide band method. They are ranging systems that are used to measure the distance between a broadcast station sending the signals and the mobile units receiving the signals.

\subsection{Ultrasound-radio frequency method of ranging}

The ultrasound-radio frequency method uses two kinds of signals. One of them is an ultrasound signal and the other is a radio frequency signal. The ultrasound and radio frequency signals are broadcasted by the broadcast stations simultaneously. The radio frequency broadcast is necessary for triggering a counter within the mobile sensor units and the ultrasound broadcast is necessary for stopping the counter. The radio frequency signal will be received by the mobile sensor units with a negligible amount of time delay compared to the ultrasound signal regarding the dimension of the area because it will travel with the speed of light. That is the time delay of the radio frequency signal can be assumed to be $T_{R F} \cong 0 \mathrm{~s}$. However, the ultrasound signal will reach at any mobile sensor unit, $b_{i}$, with a delay of $T_{U S_{i j}}(d)>0 \mathrm{~s}$ for $i=1,2, \ldots, n$ and $j=1$, $2, \ldots, m$ where $n$ is the number of mobile sensor units and $m$ is the number of broadcast stations. As seen, time of delay $T_{U S_{i j}}(d)$ is a function of the distance $d$. Normally, $m=2$. The time delay is also called as Time-of-Flight. As soon as the radio frequency signal is received any one of the mobile units, it immediately starts a counter inside the mobile sensor unit to count until the ultrasound signal is received. When an ultrasound signal is received, the counter will stop counting and store the counted value. This value is directly proportional to the time delay $T_{U S_{i j}}(d)>0 \mathrm{~s}$ and therefore the corresponding mobile sensor unit would determine its distance from the related broadcast station using this value. The same mobile sensor unit will do the same counting function for the second broadcast station and will also determine its distance from the second broadcast station and so on so forth until all broadcast stations are processed. Each of the mobile sensor units will do the following calculations to find the spatial distance, $d$, between itself and broadcast stations.

$r_{i j}=v_{\text {sound }} \times T_{U S_{i j}} \quad(i=1,2, \ldots, n \quad$ and $\quad j=1,2, \ldots, m)$

where $r_{i j}$ is the radius for the circle centered at $j$ th broadcast station and passing through the $i$ th mobile sensor unit.

As soon as every mobile sensor unit determines its coordinate of location, each mobile sensor unit will broadcast its coordinates. The broadcast, receive and motion processes are carried out according to a time division multiple access (TDMA) method described in the following.

\subsection{Time division multiple access (TDMA) method}

In this method, a sequence of actions in time is defined. Each action takes place in time slots in a time frame, $T_{F}$. The time frame repeats itself periodically. The structure of one time frame is shown in Fig. 2. The periodicity of the frame is $\left(t_{0}+n T_{f}\right)$ Main broadcast station broadcasts radio frequency and ultrasound signals at these instances. Since the radio frequency signal can reach at the mobile sensor units with negligible delays, we considered that it can be received by all mobile units at this instant. However, ultrasound signals can reach at the mobile sensor units with different time delays depending on the distances of the mobile sensor units to the broadcasting station. The time slot for broadcast and receive processes for each broadcast station is defined as $T_{U S_{\max }}$ and is selected as the time necessary for the sound to reach at the farthest point within the area of operation.

$T_{U S_{\max }}=\frac{d_{\max }}{v_{\text {sound }}}$

The next broadcast stations will send the signals at $t_{i}$, $i=1,2, \ldots, m$. This time instant must be after the $T_{U S_{\max }}$. Now, we can define the time slots for broadcast stations as

$\Delta t_{i}=\left(t_{i}-t_{i-1}\right) \geqslant T_{U S_{\max }}, \quad i=1,2, \ldots, m$

The mobile sensor units $b_{i}^{\prime} s$ need a time to make calculations and communications among themselves and the main broadcast stations at time slots assigned to them. This time interval is defined as broadcast time, $T_{b}$. At this time interval, robots calculate their coordinates and broadcast it together with their identification information. After every robot completes their broadcasting process, they enter a time interval in which they perform their rotational and translational motions simultaneously. We define this time interval as motion time, $T_{m}$.

The TDMA frame time is then defined as

$T_{f}=m T_{U S_{\max }}+n T_{b}+T_{m}$

where $n$ is the maximum number of mobile robots acting on the region, $m$ is the number of broadcast stations. Notice that we have chosen that $\Delta t=T_{U S_{\max }}$. Now the maximum number of mobile sensor units operating in this time frame can be found as

$n_{\max }=\frac{T_{f}-m T_{U S_{\max }}-T_{m}}{T_{b}}$

Calculation time $T_{b}$ for a mobile sensor unit is depended on the clock frequency of the PIC microcontroller chosen and the total instruction length of the routine for calculation. A motion of any mobile robot is composed of a rotation with a specified angle and a one step translation after the rotation. In our application, a rotation of $90^{\circ}$ and one step translation take $20 \mathrm{~s}$. The maximum time for motion in this case is chosen as $T_{m}=40 \mathrm{~s}$. The figure represents the time for rotation of $180^{\circ}$. It includes one step translation as well. In Fig. 2, positive impulses represent signal broadcasts and negative impulses show signal receives. 


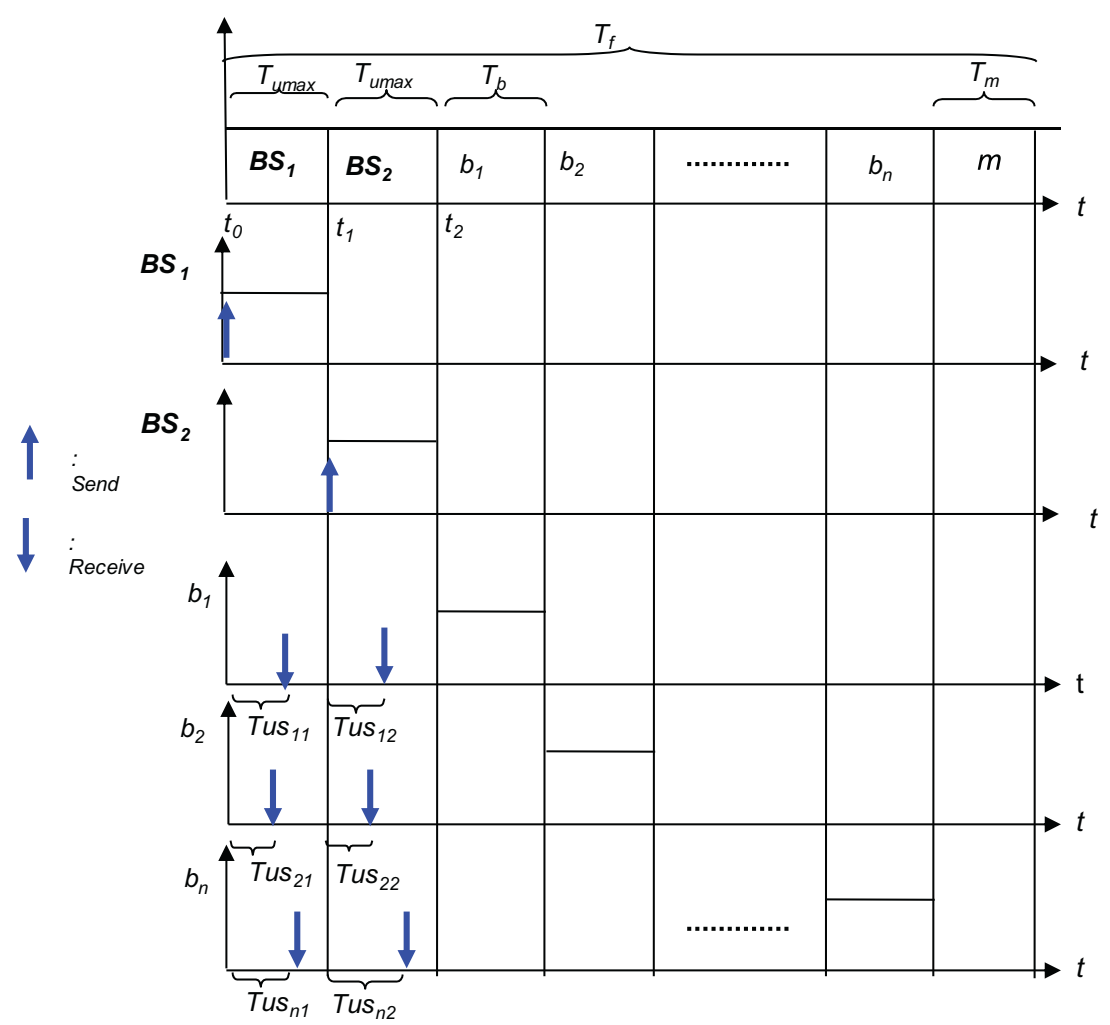

Fig. 2. TDMA frame definition for two broadcast stations broadcasting simultaneously both ultrasound and radio frequency signals. Legend: $T_{U S m a x}$ : time for sound wave to travel max. distance in the area of operation; $T_{b}$ : time for mobile sensor for calculation; $T_{m}$ : time for robot for motion; $T_{f}$ : time for TDMA frame; $B S_{j}$ : broadcast stations $(j=1,2) ; b_{i}$ : ith mobile sensor $(i=1,2, \ldots, n) ; T_{U S i j}$ : sound signal for $i$ th mobile sensor $(i=1,2, \ldots, n)$ for $j$ th broadcast station $(j=1,2)$.

\subsection{Ultra wideband radio method of ranging}

In recent years, ultra wideband (UWB) methods have received wide interest after the US Federal Communications Commission (FCC) allowed the use of unlicensed UWB communications [14]. Its advantage is mainly in the use of short-range and indoor applications. However, it is not a new concept. Since the generation of short pulses are easy, the early works on electromagnetic communications carried out by Hertz who is the famous scientist proving practically the Maxwells' equations and by Marconi who is the inventor of commercial radio used spark-gap transmitters. They are a kind of UWB radio which does not require any kind of up-conversion and down-conversion. It is a baseband communications system. An ideal UWB radio transmits an impulse function (Eqs. (6) and (7)):

$\delta(t)=\left\{\begin{array}{lll}\infty & \text { for } & t=0 \\ 0 & \text { for } & t \neq 0\end{array}\right.$

and

$\int_{-\infty}^{\infty} \delta(t) d t=1$

The Fourier transform of this signal is

$\mathfrak{F}\{\delta(t)\}=1$.
Time-domain and frequency-domain graphs of the impulse function are depicted in Fig. 3. As seen, it has a frequency spectrum of infinite bandwidth. In practice, such a signal is impossible to generate. Instead, an approximation to it can be generated. One way of obtaining a practical impulse signal is to use the Gaussian monocycle that is based on the first derivative of the Gaussian function.

$x(t)=X \frac{t}{\tau} e^{-6 \pi\left(\frac{t}{\tau}\right)^{2}}$

where $X$ is an amplitude scale factor and $\tau$ is the time constant of the pulse. The typical pulse width $\tau$ is between 0.20 and 1.50 nanoseconds. The frequency spectrum of it can be obtained by taking the Fourier transform of Eq. (9):

$X(f)=\mathfrak{F}\{x(t)\}$

The time-domain and frequency-domain graphs of the UWB signal are shown in (Fig. 4).
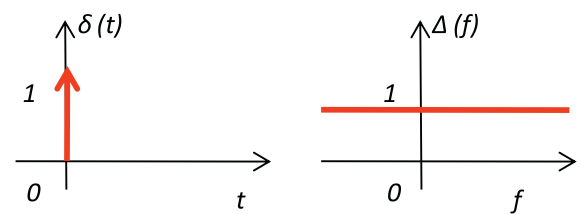

Fig. 3. Ideal impulse function and its Fourier transform. 

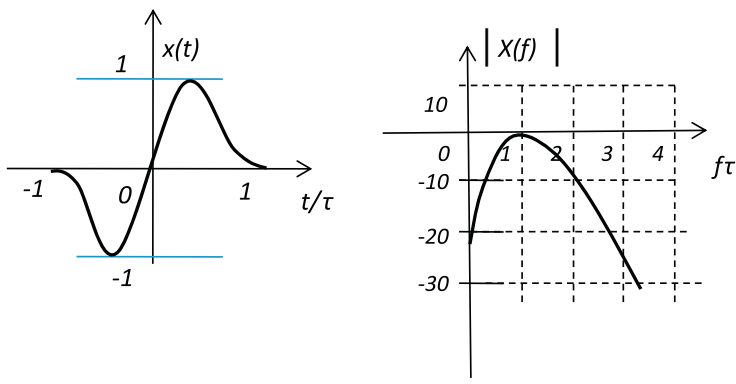

Fig. 4. A Gaussian monopulse with respect to normalized time and its frequency spectrum with respect to normalized frequency.

The time axis and the frequency axis in Fig. 4 are normalized in terms of time constant $\tau$. For $\tau=1.0$ nanosecond, this frequency axis ranges from 0 to $5 \mathrm{GHz}$. In this figure, $f_{c}=1 / \tau$ and $B W_{-10 d B}=2 / \tau$. Notice that the bandwidth of the UWB signal is greater than its center frequency. In this work, we prefer to use Gaussian monopulse as the practical impulse function since it is widely used in applications and can be implemented rather easily. The UWB signals can easily be used for geo localizations of mobile robots within a small scale environment. It is based on time of arrival of signals. A variety of techniques can be used to exchange knowledge about the absolute transmission time of the signal [9]. One of the methods is the so called "ping-pong" scheme in which there are two devices A and B having a distance between them is $d$. Device A sends a pulse, device B receives it after $t_{d}$ nanosecond, and after a certain time, $t_{B}$, replies with a signal of its own. After determining the arrival time of this pulse, device A knows the total roundtrip time of the signal between two devices. This operation can be seen in Fig. 5 . Both $A$ and $B$ can receive a pulse and retransmit it after a fixed time delay of $t_{A}$ and $t_{B}$ respectively.

We can write the expression of the received impulse signal as follows:

$r_{m}(t)=a_{d} x\left(t-t_{d}\right)+\sum_{n=1}^{k} a_{n} x\left(t-t_{n}\right)+n_{m}(t)$

where $t_{d}<t_{i}, i=1, \ldots, k$ and $\tau_{d}$ is the arrival time. $a_{d}$ is the strength of the direct path signal. $a_{i} x\left(t-t_{i}\right), i=1, \ldots, k$ are multipath signals. $k$ is unknown a priori. $n_{m}(t)$ is the noise

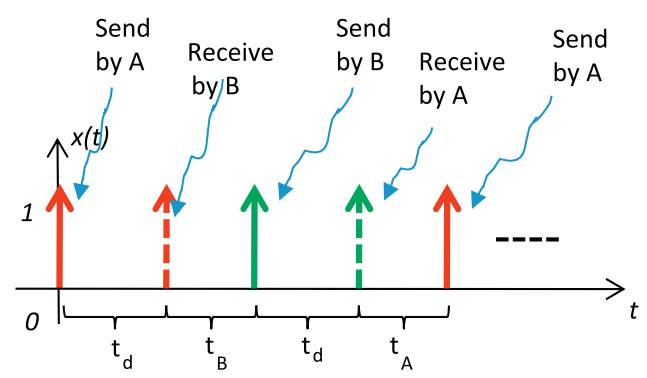

Fig. 5. The ping-pong scheme for estimation of arrival time and hence the distance between $\mathrm{A}$ and $\mathrm{B}$.

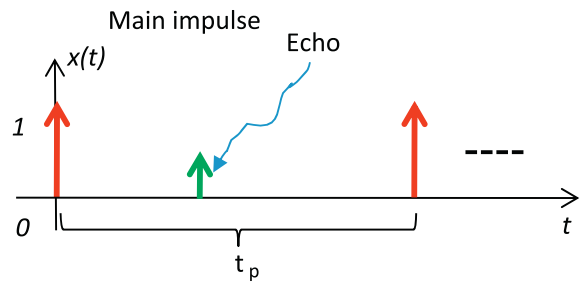

Fig. 6. Multipath effects on impulse (or UWB) radio.

that is assumed to be additive white Gaussian. Interference is assumed to be zero [13]. In indoor environments, the multipath differential delay, or delay spread, tends to be small, often on the order of nanoseconds. Since most of the time an impulse radio is not transmitting, by proper design, these echoes will arrive at the receiver in between the signaling epochs (Fig. 6). Consequently, for a receiver that is attuned to the timing of the main signal, the echoes will have no effect [12]. Note that the speed of light is $c=0.3 \mathrm{~m} / \mathrm{ns}$. So, reflections with path difference of about $1 \mathrm{~m}$ can be resolved easily by the impulse signal receiver. The upper limit on allowable path difference is determined by the pulse interval $T_{p}$. If we have direct line-of sight condition, then the multipath terms will disappear. As seen from Fig. 5 that the repetitive signals transmitted will have a period $T_{R}$ which can be written as

$T_{R}=2 t_{d}+t_{A}+t_{B}$

Or the frequency

$F=\frac{1}{T_{R}}=\frac{1}{2 t_{d}+t_{A}+t_{B}}$

Since the electromagnetic pulse travels with the speed of light $c$, arrival time to the distance $d$ is equal to $t_{d} / c$. Now, by taking into account the channel propagation time as the lower bound for the line-of-sight condition, the random contribution of multipath phenomena to propagation time may be modeled as a random variable with a positive mean $\eta$ and standard deviation $\sigma_{\tau}$ such that the probability of such variable assuming negative values is negligible. Also by assuming jitter contributions are random variables, the signal period can be written as

$T_{R n}=\frac{2 d}{c}+t_{A}+t_{B}+2 \eta+\omega_{n}$

where $\omega_{n}$ is a sequence of independent identically distributed random variables with zero mean and standard deviation $\sigma$ given by

$\sigma=\left(\sigma_{A}^{2}+\sigma_{B}^{2}+2 \sigma_{\tau}^{2}\right)^{\frac{1}{2}}$

where $\sigma_{A}$ and $\sigma_{B}$ are the standard deviations of the jitter introduced by the nodes $A$ and $B, \sigma_{\tau}$ is the standard deviation of the multipath delay contribution for each signal trip. Assuming $\sigma \ll t_{A}+t_{B}+2 \eta$ and $t_{A}, t_{B}$ and $\eta$ have properly been determined and $f$ is estimated by measuring the number of pulse repetitions $N$ in a given time $T$ by a counter. The estimators for frequency and distance can be written in a straight-forward manner as 
$\hat{f}=\frac{N}{\tau}$

$\hat{d}=\frac{c}{2\left(\frac{1}{f}-t_{A}-t_{B}-2 \eta\right)}$

Notice that $\hat{d}$ is an unbiased estimator of the distance $d$. It is shown in [13] that the relation between the CramerRao lower bounds $C R L B_{f}$ and $C R L B_{d}$ on the estimation of $\mathrm{f}$ and $\mathrm{d}$ are as follows:

$C R L B_{f}=\frac{\sigma^{2} c^{2}}{N \eta^{4}}$

$C R L B_{d}=\left(\frac{C^{2}\left(\frac{2 d}{c}+t_{A}+t_{B}+2 \eta\right)^{4}}{4 C R L B_{f}}\right)$

\subsection{Direction finding}

Mobile sensors move under two main attraction forces. One of them is the attraction force between $b_{i}$ and all other $b_{j}^{\prime} s, j=1, \ldots, k$ which are the members of the same team. This force is a vector whose entries are the genetic background information and geographical location of the MS. It is communicated among the team members. The second attraction force is the gradient vector of the potential field at the location of the $b_{j}^{\prime}$ s. The Mobile sensor can move in the direction of resultant vector that is the linear combination of the vector representing the direction of its team members and the vector representing the direction of the gradient of the potential field. Therefore, the direction of motion is the resultant vector of these two forces mentioned above. Each $b_{i}$ calculates a direction vector $\underline{d}_{i}$ pointing the team members who are broadcasting the information composed of their genetic information and the coordinates of their locations. The MS receiving the information calculates all the direction vectors towards its team members. The resultant direction vector is the sum of all the vectors $\underline{d}_{j}$ 's, that is,

$\underline{d}_{M S_{i}}=\sum_{j=1}^{k} \underline{d}_{j}$

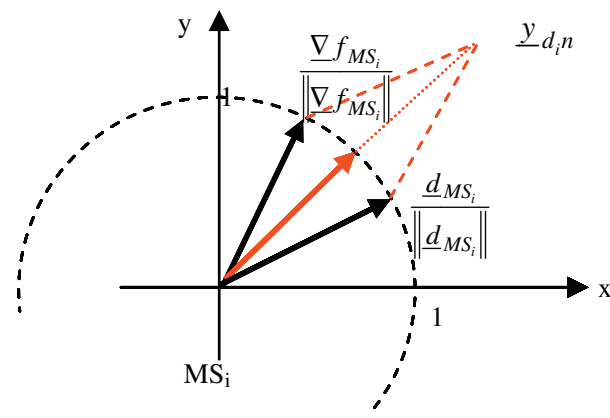

Fig. 7. Determination of direction of motion of any MS.

where $k$ is the number of team members. Each MS determines another direction vector, that is the gradient of $f(x, y)$ representing the direction of maximum increase of the potential field sensed by the gradient sensors.

$\underline{\nabla} f_{M S_{i}}=\frac{\partial}{\partial x} f(x, y) \underline{x}+\frac{\partial}{\partial y} f(x, y) \underline{y}$

The direction of motion of the $i$ th MS is the resultant vector of $\underline{d}_{M S_{i}}$ and $\nabla f_{M S_{i}}$ as

$\underline{y}_{d_{i}}=\underline{d}_{M S_{i}}+\underline{\nabla} f_{M S_{i}}$

We can interpret the behavior with unity vectors. So, we can normalize the direction vectors as seen in Fig. 7.

The gradient vector $\nabla f_{M S_{i}}$ can be determined by means of the subsystem given in Fig. 8. The subsystem is composed of a mechanism made up of eight magneto-resistive sensors $\mathrm{SN}_{k}^{\prime} s$ that are arranged in a form of gradient mask for the gradient vector $\nabla f_{M S_{i}}$ determination.

The circuit in Fig. 8 is based on a simple PIC16f84A controller. The voltages of each of the sensors in the gradient operator are converted into a eight-bit digital value by the analog to digital converters ADC0832s. The PIC controller reads the output data of each $A D C$ and calculates the gradient vector $\nabla f_{M S_{i}}$. The PIC controller also gets the genetic background data from the other $b_{i}^{\prime} s$ and calculates the direction vector $y_{d_{i}}=\underline{d}_{M S_{i}}+\underline{\nabla} f_{M S_{i}}$ and operates the dc motors driving the $b_{i}^{\prime} s$ to the direction of $\underline{y}_{d_{i}}$ by a rotational

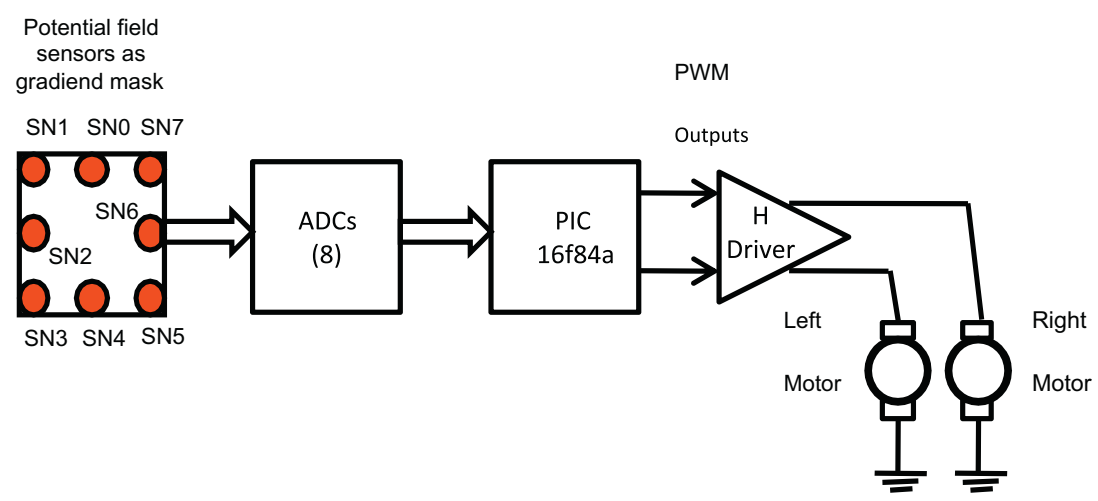

Fig. 8. The mechanism for the gradient vector $\nabla f_{M S_{i}}$ determination. Eight magneto-resistive sensors $\mathrm{SN}_{k}^{\prime} s$ are arranged in a gradient mask to detect the direction of the gradient vector in the magnetic field at the location of the mobile unit. 


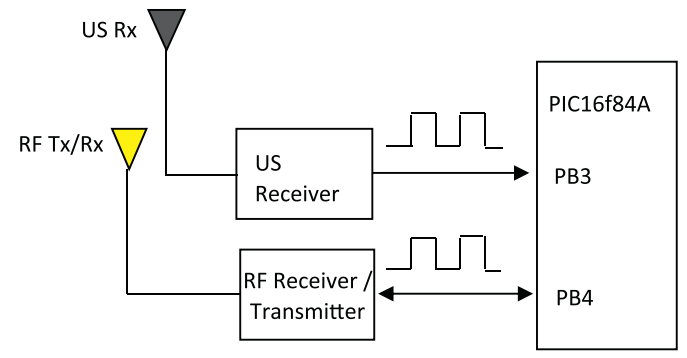

Fig. 9. Radio frequency transmitter and receiver and ultrasound receiver subsystems.

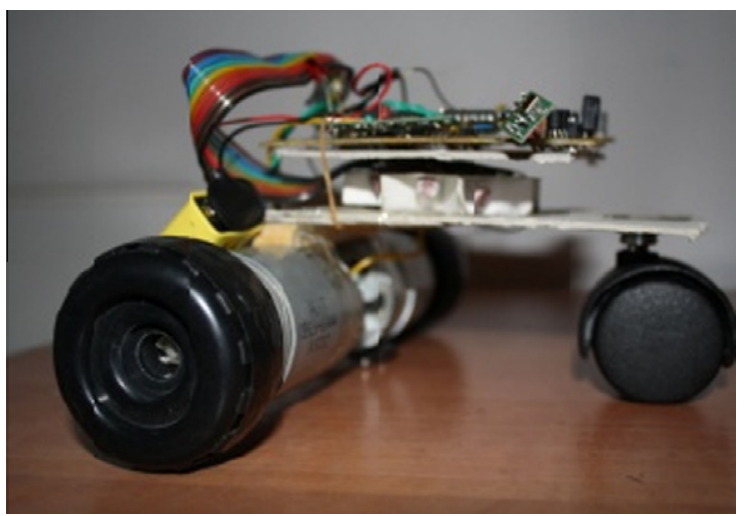

Fig. 10. A simple MS.

motion. After the rotation is completed, the $b_{i}^{\prime} s$ take a onestep forward translation in that direction. All $b_{i}^{\prime} s$ perform the same behavior simultaneously at the time slot assigned for the motion. Then the TDMA cycle starts again. The block diagram for ultrasound and RF communications mounted on $b_{i}^{\prime} s$ are given in Fig. 9. The RF receiver receives the RF pulse sent by the broadcast stations. This is the start pulse of the counter implemented in the PIC microcontroller that determines the time elapsing during the travel of the sound signal sent from the central station (and secondary station) to the mobile sensor. The ultrasound pulses received are used to stop the counter. The resulting value is proportional to the radius of the circle passing through the mobile sensor. A photograph of one simple mobile sensor is shown in Fig. 10. It is a three-wheel sumo type robot vehicle. It is an experimental system just developed to prove the concept.

\section{Application study}

The application study covers the simulations and practical applications. Since practical applications are limited to do because of the laboratory environment, we support the work by simulations. We expect that the simulation results will be helpful for the proof of the approach proposed here.

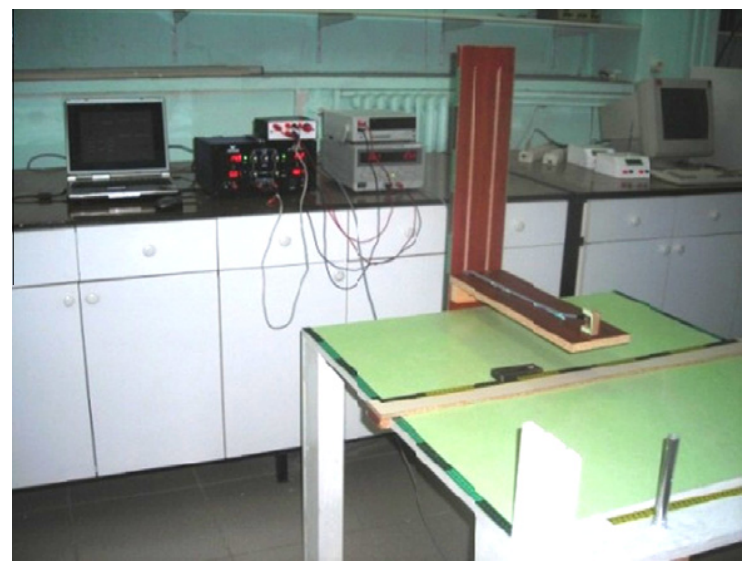

Fig. 11. The test setup.

\subsection{Test setup}

The test setup is shown in Fig. 11. A KMZ51 anisotropic magnetoresistive sensor used for measurement purpose is mounted on a platform that can perform a 3-dimensional motion. The output voltage of the sensor is converted into the digital form by a 24-bit analog-to digital converter (AD7714). The data that is digitized by the ADC is transferred to the computer through a parallel port. The data processed is used for simulations.

\subsection{KMZ51 anisotropic magnetoresistive sensor (AMR)}

The KMZ51 is an extremely sensitive magnetic field sensor, employing the magnetoresistive effect of thin-film permalloy. The sensor contains one magnetoresistive Wheatstone bridge and integrated compensation and set/ reset conductors. The integrated compensation conductor allows magnetic field measurement with current feedback loops to generate an output that is independent of drift in sensitivity. With the integrated set/reset conductor the orientation of sensitivity may be set or changed (flipped). A short current pulse on this conductor is needed to recover (set) the sensor after exposure to strong disturbing magnetic fields. A negative current pulse will reset the sensor to reversed sensitivity. By use of periodically alternated flipping pulses and a lock-in amplifier, output will become independent of sensor and amplifier offset.

\subsection{Readings of sensor voltage}

The sensor used produces two voltages $-V_{\text {out }}$ and $+V_{\text {out }}$ corresponding to the magnetic field sensed. Since the value of the earth magnetic field is not higher than the value of $H_{\text {limit }}$ which is the boundary magnetic field value, the two output voltage variations of the sensor is linear. In this work, the registers of the AD7714 are programmed through a parallel port of the computer. 


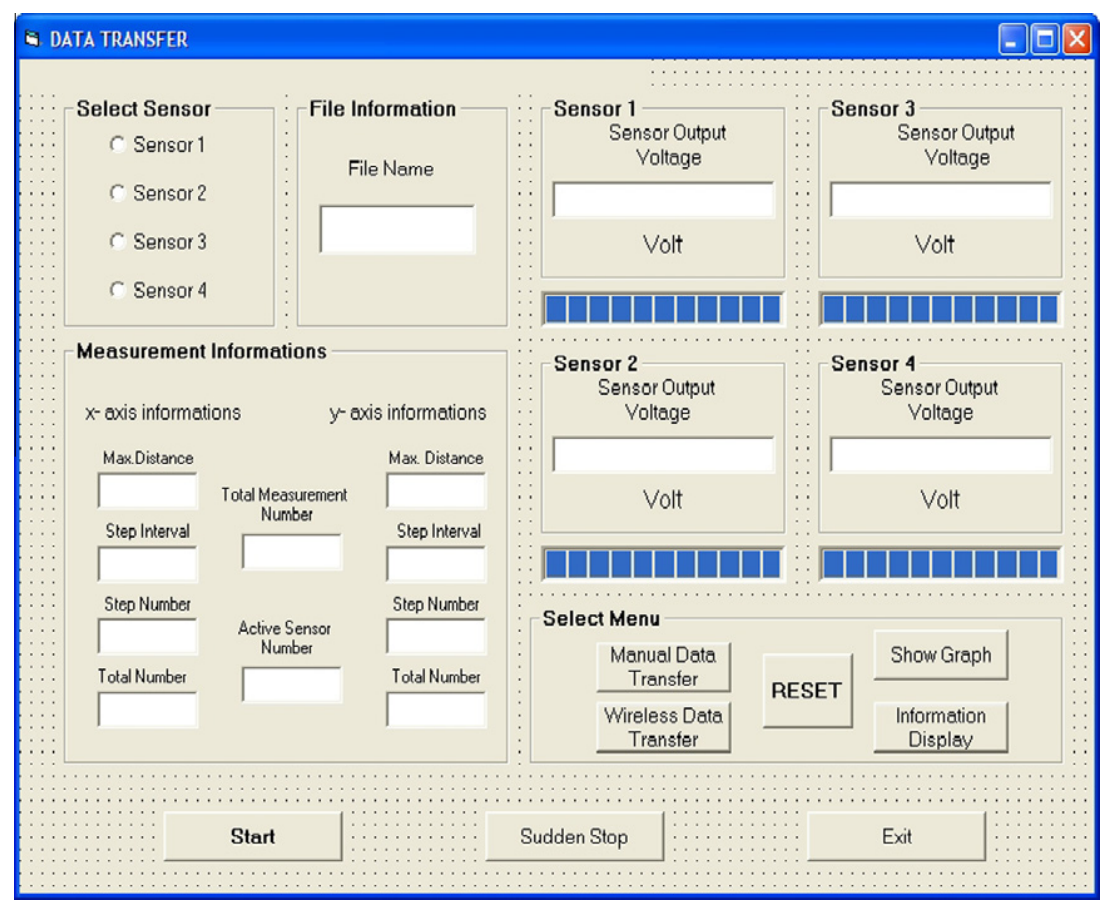

Fig. 12. The man-machine interface of the software developed here.

Table 1

Behaviour table for four MSs in two teams.

\begin{tabular}{|c|c|c|c|c|c|c|c|c|}
\hline \multirow[t]{2}{*}{ Motion } & \multicolumn{2}{|c|}{ MS $\mathbf{1}_{\mathbf{1}}$ (Team 1) } & \multicolumn{2}{|c|}{$\mathbf{M S}_{\mathbf{2}}($ Team 1) } & \multicolumn{2}{|c|}{$\mathbf{M S}_{\mathbf{3}}($ Team 2) } & \multicolumn{2}{|c|}{$\mathbf{M S}_{\mathbf{4}}($ Team 2) } \\
\hline & Direction & $\begin{array}{l}\text { Rotation and } \\
\text { coordinates }\end{array}$ & Direction & $\begin{array}{l}\text { Rotation and } \\
\text { coordinates }\end{array}$ & Direction & $\begin{array}{l}\text { Rotation and } \\
\text { coordinates }\end{array}$ & Direction & $\begin{array}{l}\text { Rotation and } \\
\text { coordinates }\end{array}$ \\
\hline Step 0 (initial state) & & $\begin{array}{l}0^{\circ} \\
(22.30)\end{array}$ & & $\begin{array}{l}0^{\circ} \\
(41.16)\end{array}$ & & $\begin{array}{l}0^{\circ} \\
(75.16)\end{array}$ & & $\begin{array}{l}0^{\circ} \\
(93.16)\end{array}$ \\
\hline Step 1 & & $\begin{array}{l}0^{\circ} \\
(22.30)\end{array}$ & & $\begin{array}{l}0^{\circ} \\
(41.16)\end{array}$ & & $\begin{array}{l}0^{\circ} \\
(75.16)\end{array}$ & & $\begin{array}{l}0^{\circ} \\
(93.16)\end{array}$ \\
\hline Step 2 & & $\begin{array}{l}27^{\circ} \\
(22.33)\end{array}$ & & $\begin{array}{l}0^{\circ} \\
(41.19)\end{array}$ & & $\begin{array}{l}0^{\circ} \\
(75.19)\end{array}$ & & $\begin{array}{l}0^{\circ} \\
(93.19)\end{array}$ \\
\hline Step 3 & & $\begin{array}{l}17^{\circ} \\
(23.35)\end{array}$ & & $\begin{array}{l}0^{\circ} \\
(41.20)\end{array}$ & & $\begin{array}{l}0^{\circ} \\
(75.20)\end{array}$ & & $\begin{array}{l}0^{\circ} \\
(93.20)\end{array}$ \\
\hline Step 4 & & $\begin{array}{l}30^{\circ} \\
(24.35)\end{array}$ & & $\begin{array}{l}0^{\circ} \\
(41.21)\end{array}$ & & $\begin{array}{l}0^{\circ} \\
(75.21)\end{array}$ & & $\begin{array}{l}0^{\circ} \\
(93.21)\end{array}$ \\
\hline Step 5 & & $\begin{array}{l}30^{\circ} \\
(25.35)\end{array}$ & & $\begin{array}{l}17^{\circ} \\
(43.23)\end{array}$ & & $\begin{array}{l}17^{\circ} \\
(77.22)\end{array}$ & & $\begin{array}{l}0^{\circ} \\
(93.22)\end{array}$ \\
\hline Step 6 & & $\begin{array}{l}22^{\circ} \\
(26.34)\end{array}$ & & $\begin{array}{l}27^{\circ} \\
(44.24)\end{array}$ & & $\begin{array}{l}0^{\circ} \\
(78.24)\end{array}$ & & $\begin{array}{l}0^{\circ} \\
(93.23)\end{array}$ \\
\hline Step 7 & & $\begin{array}{l}-19^{\circ} \\
(28.33)\end{array}$ & & $\begin{array}{l}30^{\circ} \\
(45.25)\end{array}$ & & $\begin{array}{l}0^{\circ} \\
(80.26)\end{array}$ & & $\begin{array}{l}0^{\circ} \\
(93.24)\end{array}$ \\
\hline
\end{tabular}

\subsection{The control program of measurement system}

During the scanning operation, the sensor output is electronically connected to the DC analog input at the control circuit. Thus, at each step of $1 \mathrm{~cm}$, sensor output voltage is transferred to the computer as a 24-bit resolution. The data collected are plotted as a two dimensional graphical image. 


\subsection{The detection processes}

The detection processes are implemented in the Visual $\mathrm{C}++$ environment. The software developed is used for both simulations and also the monitoring purposes of the practical applications as a parallel animation based on the data sent by the mobile sensors during their operations within the potential field. For simulation purposes, the software implements both detection processes and the motion process.

\subsection{Navigation within the potential field environment}

Experiments are performed using two different potential fields, namely a lightened environment and a magnetic environment. The central computer is used to monitor the operations. The overall process can be described as follows: The mobile sensor units are located within the field in a random manner. The mobile sensors determine their positions by use of the established local navigation and positioning system periodically within their time-slots and transfer this coordinate information and direction vectors together with their background classification vector to the central computer. The teams are given different colors by the monitoring software running in the central computer in order to facilitate the monitoring task. The information mentioned above is sent to the central computer for each mobile sensor in its appropriate time-slot. The mobile sensors are free to move around the region based on two types of information. One is the attraction force of the field distribution which is the gradient vector obtained by the gradient mask consisting of field sensors. Second is the attraction force of the team members. The movements of mobile sensors are monitored and observed step by step by the central computer. Although the number of practical units is limited, they behave as it is expected. They can sense the field appropriately and they move to the source of the field without any difficulty in an expected manner.

\section{Experiments and results}

In this section, experiments and their results are presented. A mobile sensor network ( $\mathrm{SN}$ ) is established in an indoor environment. The behavior of the mobile $\mathrm{SN}$ is investigated within the field. The field a minefield where a magnetic anomaly is created by buried mines.

\subsection{Simulations}

We developed a software program to carry out simulations. It is used to collect the sensed data and monitor the motion of the mobile sensor network. Fig. 12 shows the man-machine interface of the software developed here. Main reason for carrying out simulations is to support the practical applications because we have limited sources for practical applications. The second reason is that a simulation environment can give a flexible application domain with a reach set of virtual systems and without any cost and a need to prepare a testing laboratory. It is possible
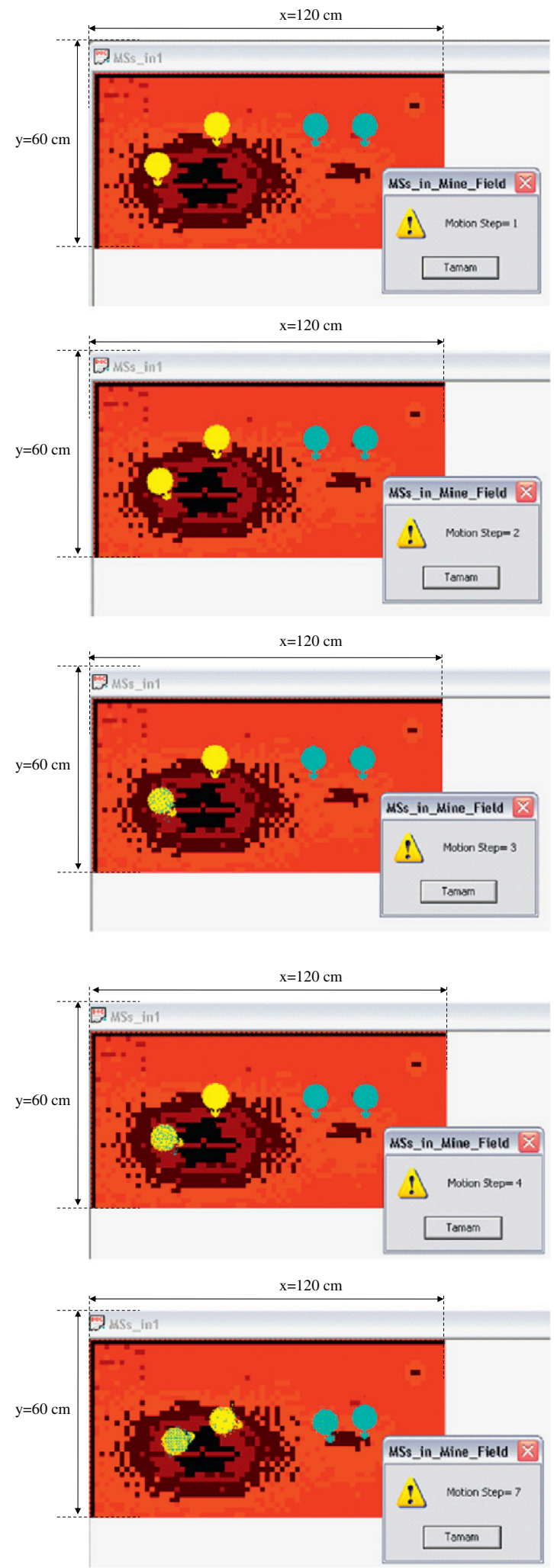

Fig. 13. Snapshots of motions of mobile sensor units during detection process. It shows initial, intermediate and final positions of the mobile sensor units during their operations. 


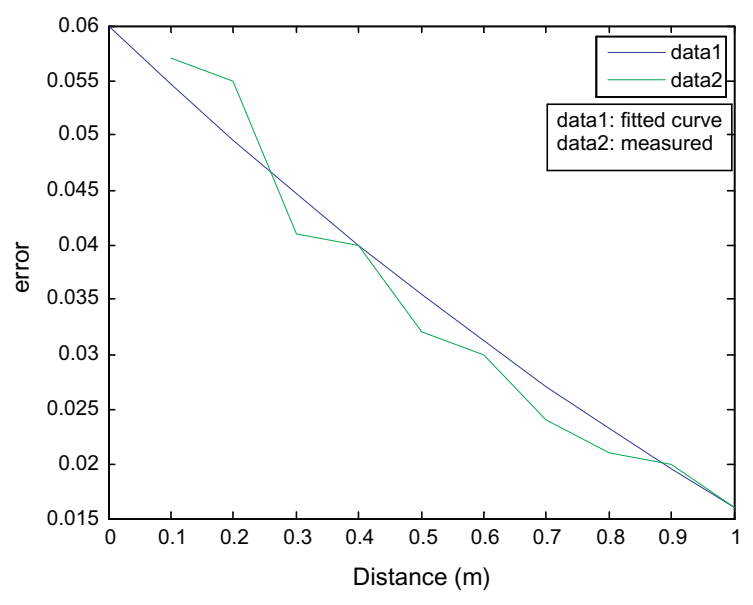

Fig. 14. Errors with respect to distances between the beacon stations.

to try a wide variety of cases and make it possible to get a lot of measurements. By the animation capability of the software, the process and the results of any practical test can be seen beforehand. Magnetic field used has anomalies caused by one anti-tank mine, one anti-personnel mine, and one ferromagnetic objects placed under the test bed. The magnetic field is read by the scanner and automatically transferred to the central computer.

The mobile sensors start their motions by sensing the magnetic field at their locations and begin to communicate with each other using the TDMA method of access. Fig. 13 illustrates snap shots of the animation. The mobile sensors start their motions at randomly selected positions in the area. They approach gradually to the central points of the magnetic anomalies. During their motions, they determine their positions with respect to the reference coordinate frame established within the region.

Table 1 summarizes the coordinate determination process.

The angular velocity of the mobile sensors during the rotational motion is $\omega=\frac{\pi}{40} \mathrm{rad} / \mathrm{s}$.

\subsection{Error analysis of coordinate determination process}

In this work, the error analysis covers the followings: (1) The measurement error for the distance between the broadcast stations functioning as the beacon stations; (2) The measurement error for gradient vectors; (3) The position error for US-RF method, and the position error for UWB ping-pong method.

The errors with respect to distances between the beacon stations are plotted as in Fig. 14. Notice that error can be reduced if the distance is increased.

The position error for gradient method is plotted as seen in Fig. 15. This represents that the error in position is fairly small.

For US-RF positioning system, and the UWB ping-pong method, the estimated frequencies and distances are obtained and they are plotted as a graph with frequency versus distance. The graph related to the UWB method is plotted as seen in Fig. 16a. The graph for US-RF method

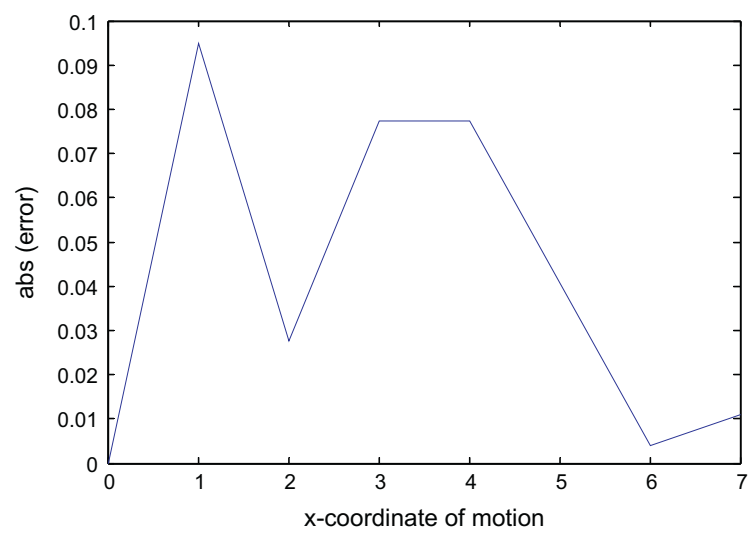

Fig. 15. The position error for the gradient method.
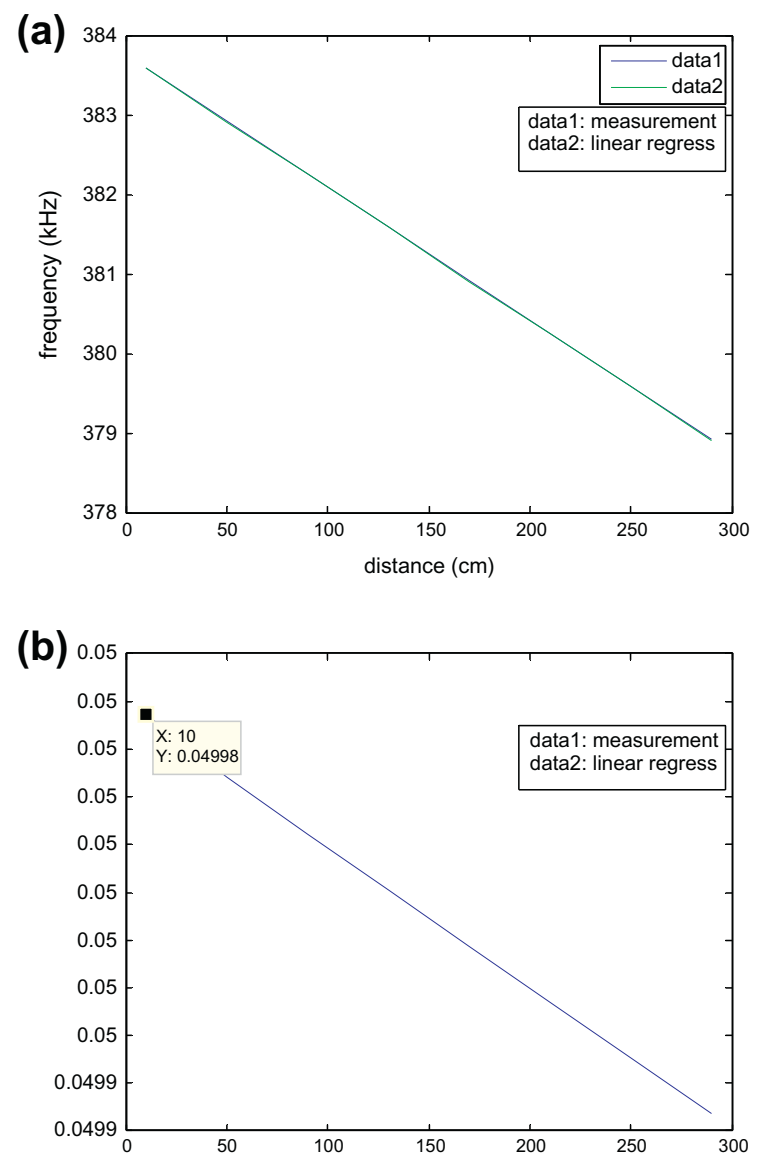

Fig. 16. Plots of measurement and fitted values for frequency versus distance (a) for UWB method; (b) for US-RF method.

plotted as frequency versus distance is given in Fig. 16b. Notice that, as it is expected, For the UWB and US-RF methods, as the distance increases, the frequency decreases.

We plotted the error against the number of measurements as shown in Fig. 17. This is the probability distribu- 


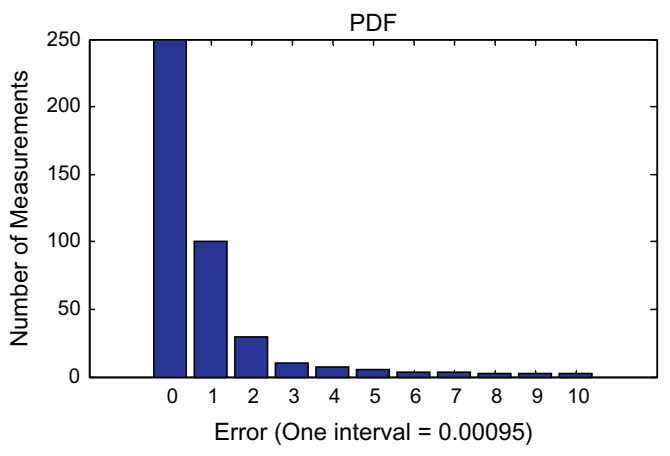

Fig. 17. Probability distribution function of the position error for US-RF and UWB ping-pong methods.

tion function (pdf), $f(|\varepsilon|)$ for the error. In order to obtain this pdf, we determined the range from $\min (|\varepsilon|)=0$ to $\max$ $(|\varepsilon|)=\varepsilon_{m}$. We subdivide the range $\left(0, \varepsilon_{m}\right)$ into $k$ equal stripes as $\varepsilon_{s}=\frac{\varepsilon_{m}}{k}$. We collected data for each stripe and we plotted the number of measurement versus error as shown in Fig. 17.

Notice that the pdf can be approximated as an exponential function. The normalized pdf is

$f(|\varepsilon|)=e^{\frac{-(|\varepsilon|-\mu)}{\beta}}$

Where the location parameter $\mu=0$ and the scale parameter $\beta=1.66$. Since the interval at the horizontal axis is 0.00095 , actual scale parameter is $\beta=1.66 \times 0.00095$ $=0.0015833$. It represents a very small variance. Therefore, we can conclude that, both of the methods can give reasonable performance. The conclusion on the results is that both of the systems can be used reliably for a local positioning system in indoor or small-scale applications of mobile sensor networks.

\section{Conclusion}

In this work, we investigated the autonomous coordinate determination of mobile sensors acting on a region where a potential field distribution is available. The practical field is a magnetic field disturbed by anti-tank and/or anti-personnel mines. The work is motivated by the decision of the authorities to clean the mines buried along a border. The aim is to detect the source of the magnetic anomaly by means of autonomously moved robotic units. The region where the mobile sensors act is either an indoor environment or a small scale outdoor environment. They sense the variations of the magnetic anomalies and tend to go in the direction of maximum decrease of the field changes. They communicate their positions among the other mobile units and they constitute a team in the region. The team swarm into the magnetic anomaly measured as the vertical component of magnetic field by sensitive sensors, namely KMZ51 anisotropic magnetoresistive sensors. The coordinate frame is made up of at least two broadcast stations acting as the beacon stations. The main broadcast station has a computer that controls the navigation process and displays the region of operation for monitoring purposes. The coordinates are determined by use of a technique called US-RF method and the results are compared with the ultra wideband ping-pong method. The operations of the units are exercised and performances are tested. Mainly, four kinds of experiments are carried out. (1) effect of inter-station distance to measurement errors; (2) errors coming from the gradient descent operations; (3) performance of US-RF coordinate determination method; (4) performance of UWB ping-pong method. The measurements are done in line-of-sight conditions. In that case, there is no multipath effects to received signals, only there might be jitter effects for the estimation performance. It is seen that coordinate errors decreases as the inter-station distance is increased. However, the error is so small that in a practical application three of the beacon stations can be mounted on the same robotic unit. This considerably facilitates the establishment of a coordinate reference frame in the operation region. The gradient descent operations may cause some placement errors in the motions of the mobile units. These errors are the results of big pixel definition and also the slippage of the mobile units during rotational motion. For small robotic units, it is seen that the errors of this kind can be tolerable. To see the level of the error against the number of measurements, the probability distribution function is obtained for hundreds of trials. I can be approximated as an exponential function with a location parameter of $\mu=0$ and the scale parameter of $\beta=0.0015833$. It represents a very small variance. Therefore we can conclude that, the method can give reasonable performances.

\section{References}

[1] S. Nazlibilek, O. Kalender, Y. Ege, Mine identification and classification by mobile sensor network using magnetic anomaly, IEEE Trans. Instrum. Meas. (99) (2010) 1-9.

[2] S. Gezici, Z. Tian, G.B. Giannakis, H. Kobayashi, A.F. Molisch, H.V. Poor, Z. Sahinoglu, Localization via ultra-wideband radios: a look at positioning aspects for future sensor networks, IEEE Signal Process. Mag. 22 (4) (2005) 70-84.

[3] I.A.R. Ashokaraj, P.M.G. Silson, A. Tsourdos, B.A. White, Robust sensor-based navigation for mobile robots, IEEE Trans. Instrum. Meas. 58 (3) (2009) 551-556.

[4] Hüseyin Akcan, Vassil Kriakov, Hervé Brönnimann, Alex Delis, GPSfree node localization in mobile wireless sensor networks, in: Proc. 5th ACM International Workshop on Data Engineering for Wireless and Mobile Access (MobiDE'06), in Conjunction with SIGMOD, pp. 35-42.

[5] L. Girod, D. Estrin, Robust range estimation using acoustic and multimodal sensing, in: Proc. IROS, 2001.

[6] A. Smith, H. Balakrishnan, M. Goraczko, N. Priyantha, Tracking moving devices with the cricket location system, in: MobiSys, 2004.

[7] J. Hill, D. Culler, Mica: a wireless platform for deeply embedded networks, IEEE Micro (2002).

[8] Y. Ding, C. Wang, L. Xiao, Using mobile beacons to locate sensors in obstructed environments, J. Parallel Distrib. Comput. 70 (2010) 644656.

[9] A. De Angelis, M. Dionigi, A. Moschitta, P. Carbone, A low-cost ultrawideband indoor ranging system, IEEE Trans. Instrum. Meas. 58 (12) (2009) 3935-3942.

[10] M.Z. Win, R.A. Scholtz, Impulse radio: how it works, IEEE Commun. Lett. 2 (2) (1998) 36-38.

[11] J.M. Cramer, R.A. Scholtz, M.Z. Win, Evaluation of an ultrawideband propagation channel, IEEE Trans. Antennas Propag. 50 (2002) 561570.

[12] J.Y. Lee, R.A. Scholtz, Ranging in a dense multipath environment using an UWB radio link, IEEE J. Sel. Area Commun. 20 (9) (2002).

[13] S. Haykin, M. Moher, Modern Wireless Communications, PrenticeHall, Pearson, 2005 
[14] First Report and Order 02-48, Federal Communications Commission, Washington, DC, 2002.

[15] O.A. Postolache, J.M.D. Pereira, P.M.B.S. Girao, Smart sensors network for air quality monitoring applications, IEEE Trans. Instrum. Meas. 58 (9) (2009) 3253-3262.

[16] H. Denkilkian, A. Koulakezian, R. Ohannessian, M.S. Chalfoun, M. Joujou, A. Chehab, I.H. Elhajj, Wireless sensor for continuous realtime oil spill thickness and location measurement, IEEE Trans. Instrum. Meas. 58 (12) (2009) 4001-4011.

[17] S. Nazlibilek, A. Erkmen, A fractal conductivity-based approach to mobile sensor networks in a potential field, Int. J. Adv. Manuf. Technol. 37 (7-8) (2008) 732-746 (15).
[18] S. Nazlibilek, Y. Ege, O. Kalender, A multi-sensor network for direction finding of moving ferromagnetic objects inside water by magnetic anomaly, Measurement 42 (9) (2009) 1402-1416.

[20] D. Gallo, C. Landi, N. Pasquino, Multisensor network for urban electromagnetic field monitoring, IEEE Trans. Instrum. Meas. 58 (9) (2009) 3315-3322.

[21] A. Carullo, S. Corbellini, M. Parvis, A. Vallan, A wireless sensor network for cold-chain monitoring, IEEE Trans. Instrum. Meas. 58 (5) (2009) 1405-1411.

[22] M.A. Hossain, P.K. Atrey, A. El Saddik, Learning multisensor confidence using a reward and punishment mechanism, IEEE Trans. Instrum. Meas. 58 (5) (2009) 1525-1534. 